\title{
Comparison of brain and spinal cord magnetic resonance imaging features in neuromyelitis optica spectrum disorders patients with or without aquaporin-4 antibody
}

\author{
Moli Fan ${ }^{\mathrm{a}}$, Ying Fu ${ }^{\mathrm{a}}$, Lei Su ${ }^{\mathrm{a}}$, Yi Shen ${ }^{\mathrm{a}}$, Kristofer Wood ${ }^{\mathrm{b}}$, Li Yang ${ }^{\mathrm{a}}$, Yaou Liu ${ }^{\mathrm{a}, \mathrm{c}, \mathrm{d}, *}$, Fu-Dong Shi ${ }^{\mathrm{a}, \mathrm{b}}$ \\ a Department of Neurology, Key Laboratory of Posttraumatic Neuro-repair and Regeneration in the Central Nervous System, Tianjin Neurological Institute, \\ Tianjin Medical University General Hospital, China \\ b Barrow Neurological Institute, St Joseph's Hospital and Medical Center, USA \\ c Department of Radiology, Xuanwu Hospital, Capital Medical University, China \\ d Department of Radiology and Nuclear Medicine, Neuroscience Campus Amsterdam, VU University Medical Center, Amsterdam 1007 MB, The Netherlands
}

\section{A R T I C L E I N F O}

\section{Keywords:}

Neuromyelitis optica

Aquaporin 4

Brain

Spinal cord

MRI features

\begin{abstract}
A B S T R A C T
Background: The spinal cord and brain measurements are rarely investigated in neuromyelitis optica (NMO) patients with and without antibodies to aquaporin-4 (AQP4), directly compared to multiple sclerosis (MS) patients.

Objectives: To investigate magnetic resonance imaging (MRI) features of both brain and spinal cord in NMO patients with and without antibodies to AQP4, compared with MS patients and healthy controls (HC).

Methods: We recruited 55 NMO including $30 \mathrm{AQP} 4(+)$ and 25 AQP4 (-), 25 MS and 25 HC. Brain and spinal cord MRIs were obtained for each participant. Brain lesions (BL), whole brain and deep grey matter volumes (DGMV), white matter diffusion metrics and spinal cord lesions were measured and compared among groups. Results: The incidence of BL was lower in the AQP4 (+) group than in the AQP4 (-) and MS groups (p < 0.05). In the AQP4 (+) group, there was a lower incidence of infratentorial lesions (ITL) and higher spinal cord lesions length than in the MS group ( $<<0.05)$. The thalamic and hippocampal volumes were smaller in the AQP4 (-) group and MS group than in the HC group $(\mathrm{p}<0.05)$.

Conclusions: The NMO patients with AQP4 (-) showed higher prevalence of BL, ITL, and similar spinal cord lesion length, compared to AQP4 (+), and demonstrated deep grey matter atrophy, suggesting an intermediate phenotype between that of typical MS and NMO.
\end{abstract}

\section{Introduction}

Neuromyelitis optica (NMO) is an autoimmune, inflammatory and demyelinating disease of the central nervous system (CNS). This syndrome is clinically characterized by recurrent attacks of optic neuritis and myelitis (Wingerchuk et al., 2007; Wingerchuk et al., 2006). The anti-aquaporin-4 (AQP4) antibody, which was first discovered in 2004, has been claimed to play a pathogenic role in NMO and can be used to achieve an early diagnosis (Lennon et al., 2004; Weinshenker et al., 2006). However, AQP4 antibody positivity is not universal in patients with clinically definite NMO, with reported sensitivities ranging from approximately 30\% to 90\% (CabreraGomez et al., 2009; Weinshenker et al., 2006).

In case series it has been demonstrated that lesions on brain magnetic resonance imaging (MRI) are common in NMO and showed differences between anti-AQP4 antibody positive and negative patients. A study in Cuba indicated that anti-AQP4 antibody positive NMO cases had more brain lesions than anti-AQP4 antibody negative NMO cases (Cabrera-Gomez et al., 2009). Whilst, Japanese and English investigators observed that brain lesions fulfilling the Barkhof criteria were significantly more common in patients without AQP4 antibodies (AQP4-Ab) than with autoimmune antibodies (Downer et al., 2012;

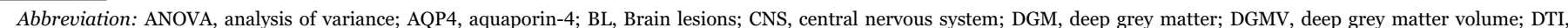

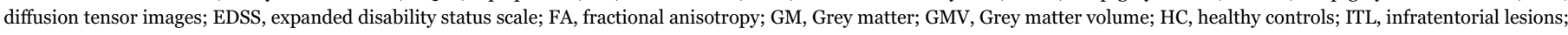

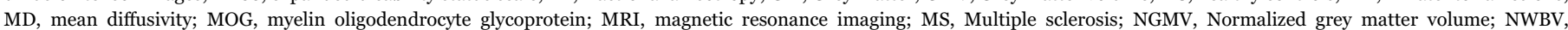

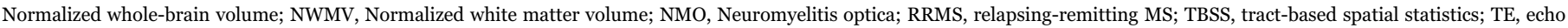
time; TR, repetition time; WBV, whole brain volume; WMV, white matter volume; WM, white matter; LETM, Longitudinally extensive transverse myelitis

* Correspondence to: Department of Neurology, Tianjin Neurological Institute, Tianjin Medical University General Hospital, 154, Anshan Road, Tianjin 300052, China.

E-mail address: yaouliu80@163.com (Y. Liu).
} 
Table1

Demographic and clinical characteristics.

\begin{tabular}{|c|c|c|c|c|c|}
\hline & AQP4 (+) & AQP4 (-) & MS & $\mathrm{HC}$ & $P$ value \\
\hline $\begin{array}{l}\text { Gender } \\
\text { (Male/ } \\
\text { Female) }\end{array}$ & $6 / 24$ & $7 / 18$ & $9 / 16$ & $7 / 18$ & $0.625^{1}$ \\
\hline Age (year) & $\begin{array}{l}48.5 \pm 11.6 \\
(22-68)\end{array}$ & $\begin{array}{l}46.4 \pm 15.6 \\
(17-66)\end{array}$ & $\begin{array}{l}43.3 \pm 9.6 \\
(24-59)\end{array}$ & $\begin{array}{l}45.9 \pm 9.2 \\
(25-63)\end{array}$ & $0.455^{2}$ \\
\hline $\begin{array}{l}\text { Disease } \\
\quad \text { duration } \\
\text { (year) }\end{array}$ & $\begin{array}{l}5.91 \pm 5.04 \\
(0-20)\end{array}$ & $\begin{array}{l}4.01 \pm 3.28 \\
(0-13)\end{array}$ & $\begin{array}{l}4.13 \pm 5.81 \\
(0.25-26)\end{array}$ & - & $0.312^{2}$ \\
\hline $\begin{array}{c}\text { Number of } \\
\text { attacks }\end{array}$ & $\begin{array}{l}3.7 \pm 3.4 \\
(1-17)\end{array}$ & $\begin{array}{l}3.3 \pm 2.0 \\
(1-11)\end{array}$ & $\begin{array}{l}2.8 \pm 1.4 \\
(2-7)\end{array}$ & - & $0.479^{2}$ \\
\hline EDSS & $\begin{array}{l}3.3 \pm 2.0 \\
(0-7)\end{array}$ & $\begin{array}{l}3.4 \pm 1.9 \\
(0-8.5)\end{array}$ & $\begin{array}{l}2.7 \pm 1.6 \\
(1-6)\end{array}$ & - & $0.427^{2}$ \\
\hline
\end{tabular}

AQP4 (+)=NMO patients seropositive for anti-aquaporin-4 antibodies; AQP4 (-) =NMO patients seronegative for anti-aquaporin-4 antibodies; MS=multiple sclerosis patients; $\mathbf{H C}=$ Healthy controls; EDSS=expanded disability status scale. P-value: groups were compared using Chi-Square test ${ }^{1}$ and ANOVA test ${ }^{2}$. Data are presented as Mean \pm SD (range)

Matsushita et al., 2010). GM atrophy and WM diffusion abnormalities in NMO patients were reported by recent studies (Chanson et al., 2013; Duan et al., 2012; Kimura et al., 2014; Liu et al., 2012; Pichiecchio et al., 2012; Rueda Lopes et al., 2012; Zhang et al., 2014), but whether these changes differ between antibody-positive and antibody-negative NMO patients is unknown. Some studies have investigated the different patterns of spinal cord lesion between anti-AQP4 antibody positive and negative patients (Jarius et al., 2012), showing that the total spinal cord lesion load was higher, as well as lesions $\geq 6$ vertebral segments, entire spinal cord involvement and lesions with Gd-enhancement (Liu et al., 2013), were more common in seropositive patients. As far as we know, there has not yet been a study investigating MRI features of both the brain and spinal cord in NMO patients (AQP4 (+) vs. AQP4 (-)), compared with well-matched MS patients and HC. Recent studies have identified antibodies against myelin oligodendrocyte glycoprotein (MOG-Abs) in NMO spectrum disorders (NMOSD) (Kitley et al., 2012, 2014; Ramanathan et al., 2016; Willison and Linington, 2012). The similarities and differences of MRI characteristics between MOG $(+)$ and (-) patients also remain unknown.

In this study, we assessed brain and spinal cord MRI characteristics including brain and spinal cord lesions, brain GM atrophy, WM diffusion measurements between AQP4-Ab-positive and AQP4-Abnegative clinically definite NMO patients, directly compared with age and gender matched MS and healthy controls. Additionally, we compared MRI characteristics of MOG (+) and (-) patients in AQP4 $(-)$ patients.

\section{Materials and methods}

\subsection{Subjects}

The study was approved by the local ethics committee and written informed consent was obtained from all patients and volunteers. We recruited 69 NMO patients (14 men and 55 women) from the
Department of Neurology in Tianjin Medical University General Hospital. The NMO diagnosis was determined according to the 2006 diagnostic criteria (Wingerchuk et al., 2006). All patients had optic neuritis and myelitis, and met 2 of the 3 supporting criteria including AQP4 antibody positivity, negative brain MRI or not meeting the diagnostic criteria for MS, and a spinal cord lesion involving at least three vertebral segments. For direct comparison with NMO patients, we selected 28 relapsing-remitting MS (RRMS) patients (9 men, 19 women) fulfilling the McDonald criteria (Polman et al., 2005) by matching them for age, gender, disease duration and EDSS score to the NMO group from an ongoing MS cohort study. 14 NMO and 3 MS cases were excluded due to incomplete clinical assessment $(\mathrm{n}=3 ; 2$ NMO, 1 MS); MRI image artifacts ( $=9$; 7 NMO, 2 MS) or the presence of other CNS diseases ( $\mathrm{n}=5$; 5 NMO, 0 MS). 55 NMO patients and 25 MS cases were entered into the final analysis. 32 Of 55 NMO patients were relapsing $\mathrm{NMO}, 30 \mathrm{NMO}$ patients were AQP4-seropositive (AQP4-Ab (+)) and 25 were seronegative (AQP4-Ab (-)). 4 patients were MOG-seropositive in the NMO AQP4-Ab (-) patients, and one patient was double positive for anti-AQP4 and MOG antibodies. All MS patients were relapsing-remitting patients and AQP4-seronegative. The main demographic and clinical characteristics, including age, disease duration, age at disease onset and expanded disability status scale (EDSS) of the patients studied are reported in Table 1. Additionally, we chose twenty-five age and gender matched healthy controls (HCs) (7 men, 18 women) with no previous history of neurological dysfunction and with normal findings on neurological examination and MRI (Table 1).

\subsection{AQP4 and MOG antibody assay}

Enhanced green fluorescent protein tagged AQP4/EGFP and MOG/ EGFP fusion protein transfected human embryonic kidney cells (HEK293) were produced as previously described (Yang et al., 2014; Zhang et al., 2015). Assays for anti-AQP4 and anti-MOG antibodies were carried out at least twice for each sample, and those that gave a positive or negative result twice were deemed to be positive or negative.

\subsection{Image acquisition}

MR imaging was performed on a 3.0 T MR system using an eightchannel phased array head coil in Tianjin Medical University General Hospital. Axial T2-weighted turbo spin echo (40 interleaved slices, repetition time $(\mathrm{TR})=6816 \mathrm{~ms}$, echo time $(\mathrm{TE})=91 \mathrm{~ms}$, echo train length $=32$, section thickness $=3 \mathrm{~mm}$, in-plane resolution $1 \mathrm{~mm}^{2}$ ) was used for lesion detection. High-resolution anatomical images were acquired using sagittal 3D T1-weighted images sequence (number of slices $=191 ; \mathrm{TR}=8.16 \mathrm{~ms} ; \mathrm{TE}=3.18 \mathrm{~ms}$; flip angle $(\mathrm{FA})=12^{\circ}$; slice thickness $=1 \mathrm{~mm}$; in-plane resolution $1 \mathrm{~mm}^{2}$ ), which was used for brain volume measurement. Furthermore we acquired 2D echo-planar diffusion tensor images (DTI) [number of slices $=69$; $\mathrm{TR}=7100 \mathrm{~ms}$, $\mathrm{TE}=61.3 \mathrm{~ms}$, slice thickness $=2 \mathrm{~mm}$, in-plane resolution $2 \mathrm{~mm}^{2} ; 50$ non-collinear diffusion gradients $\left(b=1000 \mathrm{~s} / \mathrm{mm}^{2}\right)$ and three nondiffusion-weighted images $\left(b=0 \mathrm{~s} / \mathrm{mm}^{2}\right)$ ] for measuring $\mathrm{WM}$ tract integrity. All the spinal cord imaging was acquired on the same scanner

Table 2

Clinical characteristics of 5 patients with MOG-Abs in NMO (-) patients.

\begin{tabular}{|c|c|c|c|c|c|c|c|c|c|c|}
\hline Patients & Age (year) & Sex & No. of attacks & Disease duration (year) & EDSS & Diagnosis & AQP4 Abs & MOG Abs & Other serum Abs & Treatment \\
\hline 1 & 22 & M & 2 & 6 & 2 & NMO & negative & positive & negative & $\mathrm{CS}$ \\
\hline 2 & 50 & M & 2 & 1 & 1 & NMO & negative & positive & negative & None \\
\hline 3 & 52 & M & 6 & 5 & 6 & NMO & negative & positive & negative & $\mathrm{CS}$ \\
\hline 4 & 66 & $\mathrm{~F}$ & 2 & 8 & 6 & NMO & negative & positive & negative & $\mathrm{CS}$ \\
\hline
\end{tabular}

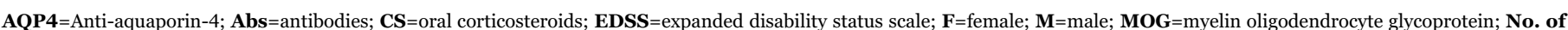
attacks=number of attacks. 

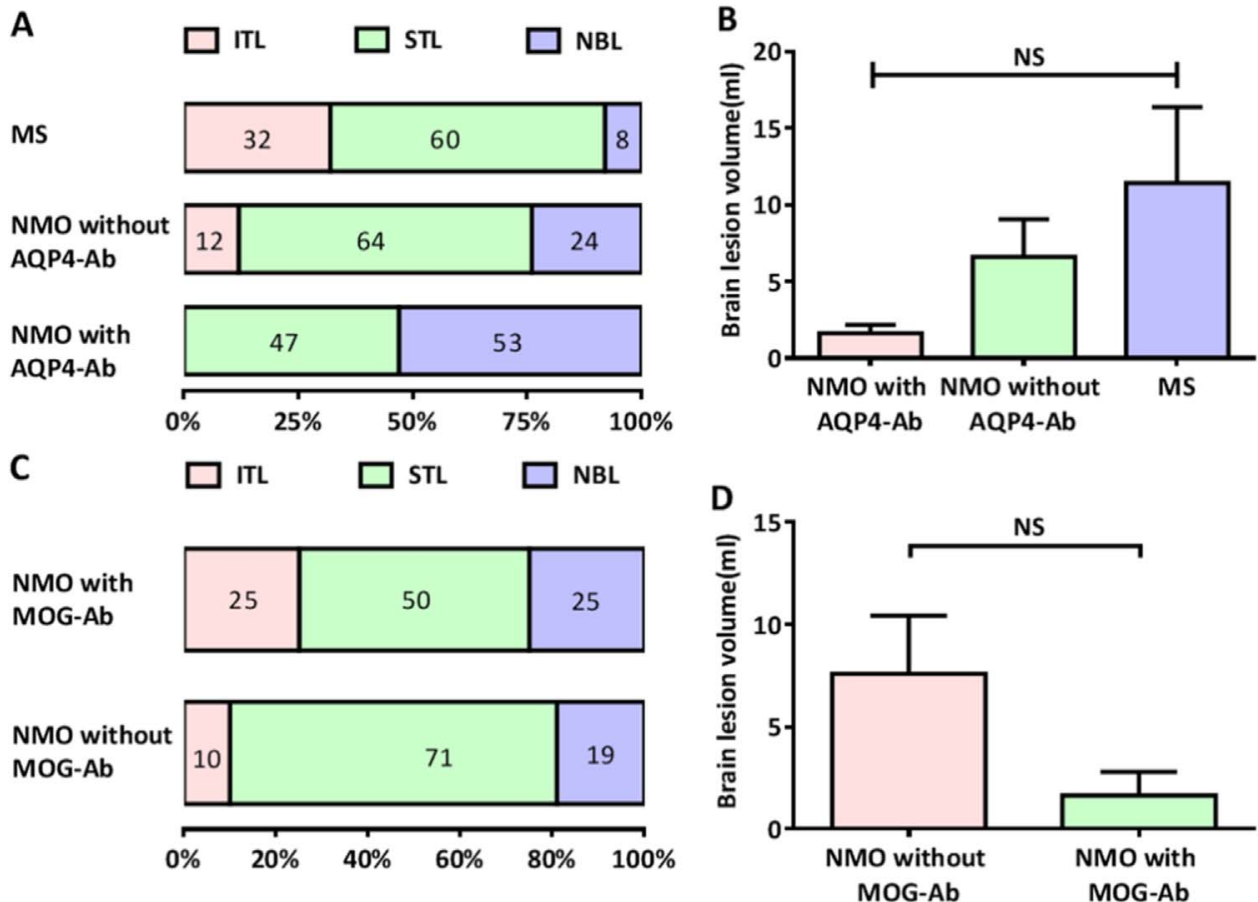

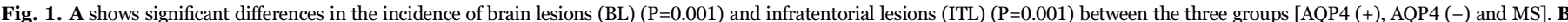

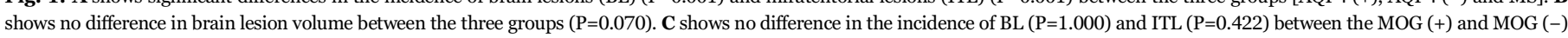
groups. D shows no difference in brain lesion volume between the MOG $(+)$ and MOG $(-)$ groups $(\mathrm{P}=0.970)$. $\mathrm{STL}=\mathrm{supratentorial}$ lesion; ITL=infratentorial lesion; NBL=no brain lesion.
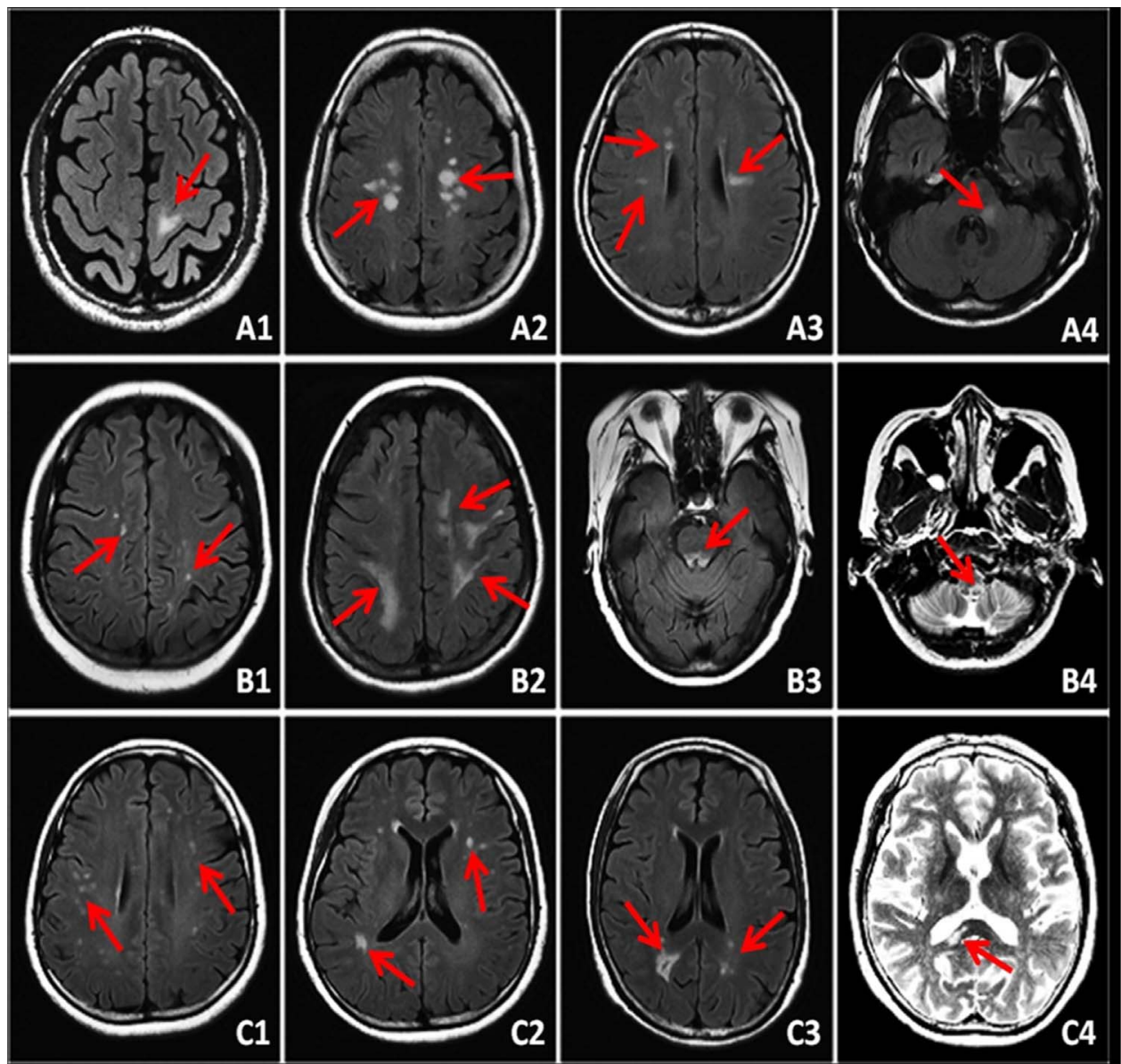

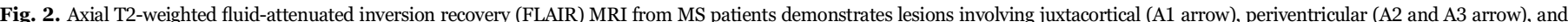

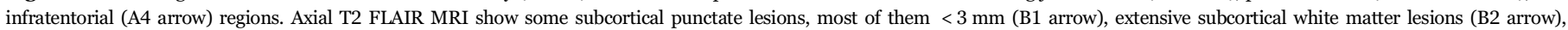

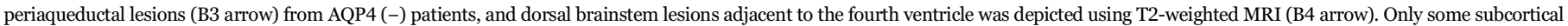
and deep white matter punctate and patchy lesions were observed using T2 FLAIR MRI (C1-C3 arrow), and corpus callosum lesions in T2 MRI (C4 arrow) from AQP4 (+) cases. 
A
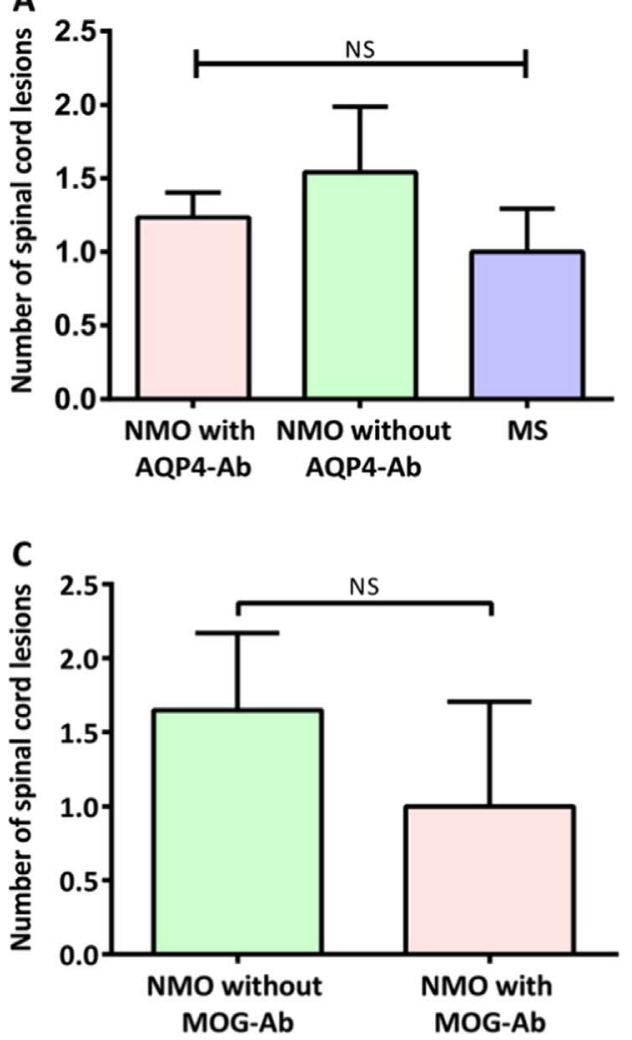

B
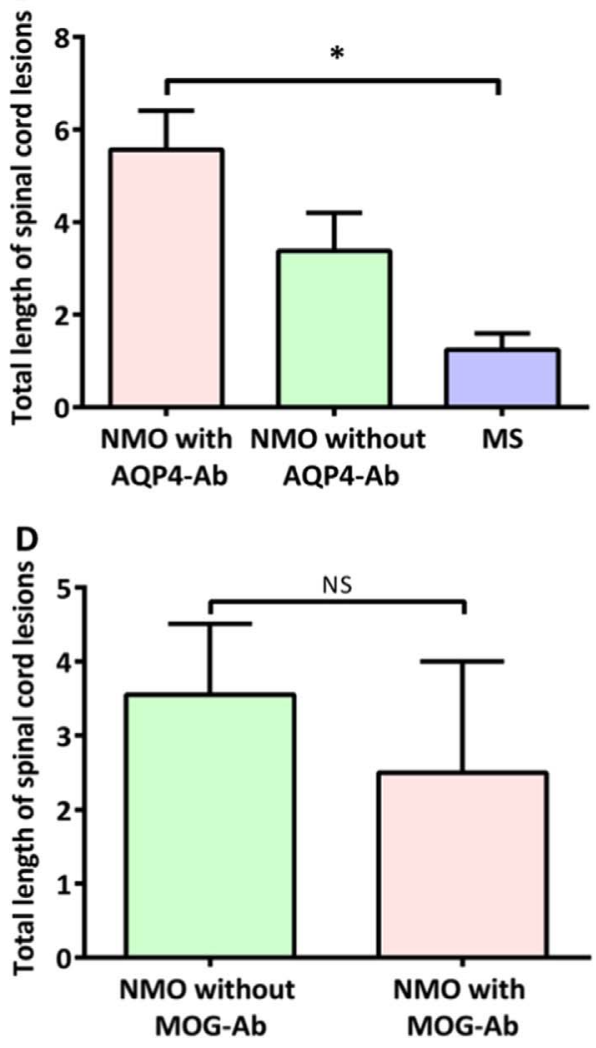

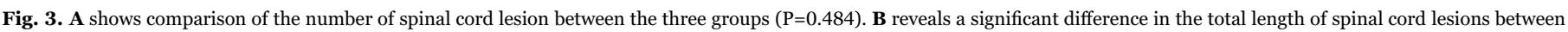

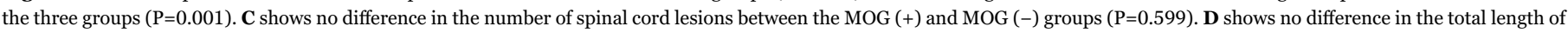
spinal cord lesions between the MOG $(+)$ and MOG $(-)$ groups $(\mathrm{P}=0.719)$.

(sagittal T1-weighted $\mathrm{SE}, \mathrm{TR}=530 \mathrm{~ms} ; \mathrm{TE}=12 \mathrm{~ms}$; slice thickness $=3 \mathrm{~mm}$; sagittal T2-weighted TSE, TR=2300 ms; TE=90 ms; slice thickness $=3 \mathrm{~mm}$; Axial T1-weighted $\mathrm{SE}, \mathrm{TR}=530 \mathrm{~ms}$; TE=13 ms; slice thickness $=4 \mathrm{~mm}$; Axial T2-weighted TSE, TR=4300 ms; TE=120 ms; slice thickness $=4 \mathrm{~mm}$ ).

\subsection{Image analysis}

Marking and measurement of hyperintense brain lesions (T2LV) on T2-weighted images was performed by an experienced neurologist using MRIcro software. Infratentorial lesions were defined as lesions in the cerebellum, pons, medulla or mesencephalon. Normalized wholebrain volume (NWBV), grey matter volume (NGMV), and white matter volume (NWMV) were computed using the 3D T1-weighted images and SIENAX (part of FSL 5.0.4) (Smith et al., 2002). In order to avoid classification of lesions as grey matter, T2 lesion masks were coregistered to the 3D T1-weighted images to perform lesion filling, using individually averaged normal appearing white matter values prior to brain tissue volume segmentation. Deep grey matter (DGM) volumes including the thalamus and hippocampus were quantified by the FIRST (also part of FSL) (Patenaude et al., 2011). The left and right volumes for each DGM structure were averaged and normalized by multiplying with the V-scaling from SIENAX. The diffusion metrics (fractional anisotropy, FA, and mean and axial diffusivity) were quantified using the diffusion-weighted images and tract-based spatial statistics (TBSS; also part of FSL). The mean FA image was thresholded at 0.2 and skeletonized. Each subject's FA and MD values were projected onto the mean FA skeleton. The average FA and MD values of the whole skeleton were calculated for each subject.

\subsection{Statistical analysis}

Analyses were performed by using SPSS software (Version 18; SPSS, Chicago, Ill). Kolmogorov-Smirnov tests were used together with visual inspection of histograms to assess normality of the variables. To compare gender and the incidence of brain lesions and infratentorial lesions between the AQP4 (+) group, AQP4 (-) group, MS group and HC group, we used the Chi-Square test. Comparisons of the demographic and MRI parameters between AQP4 (+) patients, AQP4 (-) patients, MS patients and HCs were conducted using analysis of variance (ANOVA) and the Tukey honestly significant difference test was used for post hoc comparisons. Comparisons of the MRI parameters between MOG (+) group and MOG (-) group, were performed using the $t$-test or Mann-Whitney $U$ test according to the normality of the variables. $P$ values $<0.05$ were considered statistically significant.

\section{Results}

\subsection{Demographics and clinical characteristics}

As shown in Table 1, no significant differences were observed in age and gender among the four groups (NMO AQP4-antibody positive, NMO AQP4 antibody negative, MS and HC). There were no significant differences in EDSS and disease duration between the AQP4 (+), AQP4 $(-)$ and MS patients. The clinical characteristics of the 4 NMO patients with MOG antibodies are shown in Table 2.

\subsection{Brain lesions}

The incidence of brain lesions (BL) and infratentorial lesions (ITL) showed significant differences between the three groups $(\mathrm{p}=0.001$ and $\mathrm{p}=0.001$, respectively), in which the AQP4 $(-)(\mathrm{p}=0.032)$ and MS 

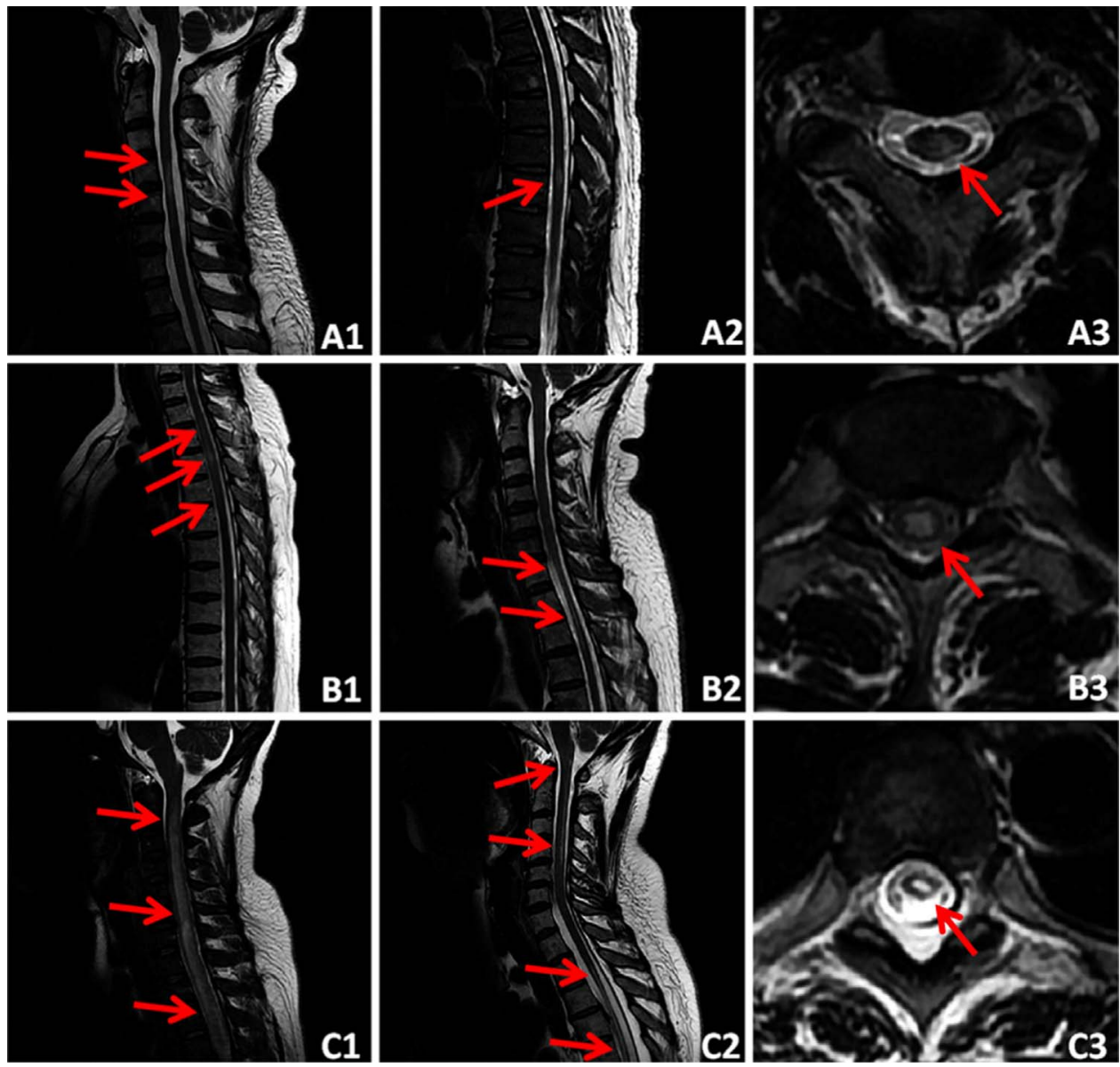

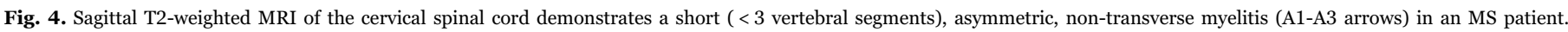

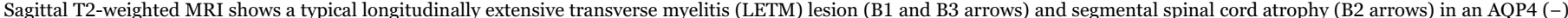

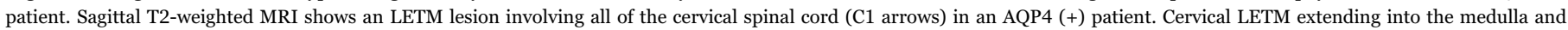
longitudinally extensive segments of spinal cord atrophy as shown by T2-weighted MRI using sagittal and axial (C2 and C3 arrows) in an AQP4 (+) patient.

groups ( $\mathrm{p}=0.000)$ showed a higher prevalence of BL than the AQP4 (+) group, no significant difference between the AQP4 (-) group and MS group ( $\mathrm{p}=0.247)$ was observed (Fig. 1A). There was a higher incidence of ITL in the MS group than in AQP4 (+) group ( $\mathrm{p}=0.001)$, but no difference was identified in NMO patients with or without AQP4-Ab $(\mathrm{p}=0.088)$ (Fig. 1A). None of the AQP4 (+) patients presented with ITL. There was no significant difference in the brain lesion volumes between the three groups $(\mathrm{p}=0.070)$ (Fig. 1B). Examples of the brain MRI images of the three groups are shown in Fig. 2. Moreover, the incidence of brain lesions (BL), infratentorial lesions (ITL) and brain lesion volume showed no differences between the MOG (+) and MOG (-) groups of the AQP4 (-) patients $(\mathrm{p}=1.000, \mathrm{p}=0.422, \mathrm{p}=0.970$, respectively) (Fig. 1C and D).

\subsection{Spinal cord lesions}

The number of spinal cord lesions was not significantly different among the three patient groups ( $\mathrm{p}=0.484$ ) (Fig. 3A). The total length of spinal cord lesions was higher in the AQP4 (+) than the MS group $(\mathrm{p}=0.000)$. No significant difference was observed between the AQP4 $(-)$ and MS group ( $\mathrm{p}=0.069)$ or the AQP4 (+) and AQP4 (-) group $(\mathrm{p}=0.193)$ (Fig. 3B). In the AQP4 (-) group, there were no significant differences in the number of spinal cord lesions and total length of spinal cord lesions between the MOG $(+)$ and MOG (-) groups $(p=0.599$ and $p=0.719$ respectively) (Fig. $3 \mathrm{C}$ and D). Examples of the spinal cord MRI images of the three groups are shown in Fig. 4.

\subsection{Brain volume}

Compared with the HCs, whole brain volume (WBV) and white matter volume (WMV) were decreased in the MS group ( $p=0.009$; $\mathrm{p}=0.001$, respectively) (Fig. $5 \mathrm{~A}$ and $\mathrm{B}$ ). The MS patients showed reduction of white matter volume (WMV) compared to the AQP4 (+) patients $(\mathrm{p}=0.005)$. Grey matter volume (GMV) showed no statistical differences among the four groups ( $\mathrm{p}=0.072)$ (Fig. 5C). In AQP4 (-) patients, WBV, GMV and WMV were similar between patients with and without MOG antibodies $(\mathrm{p}=0.266 ; \mathrm{p}=0.120 ; \mathrm{p}=0.941$ respectively) (Fig. 5D-F). Thalamic and hippocampal volumes, belonging to deep grey matter volume (DGMV), were obviously different among the four groups $(\mathrm{p}=0.039 ; \mathrm{p}=0.029)$. Thalamic and hippocampal volumes were decreased in the AQP4 $(-)$ group $(\mathrm{p}=0.023 ; \mathrm{p}=0.021)$ and the MS group ( $\mathrm{p}=0.006 ; \mathrm{p}=0.021$ ) compared to the $\mathrm{HC}$ group (Fig. $6 \mathrm{~A}$ and $\mathrm{B}$ ). However, no differences were found in the thalamic or hippocampal volumes of AQP4 (-) patients with or without MOG antibodies $(\mathrm{p}=0.505 ; \mathrm{p}=0.941$ ) (Fig. 6C and D).

\subsection{White matter tract integrity}

We used two DTI measurements, (whole brain FA and MD), to evaluate the integrity of brain white matter. There were no significant differences in either FA or MD values among three patient groups (AQP4 (+), AQP4 (-) and MS) (p=0.452) (Fig. 7A) (p=0.018) (Fig. 7B). The only significant difference was observed between the MD of MS patients and HCs $(\mathrm{p}=0.004)$ (Fig. $7 \mathrm{~B})$. There were no significant differences between the MOG (+) and MOG (-) groups of the AQP4 

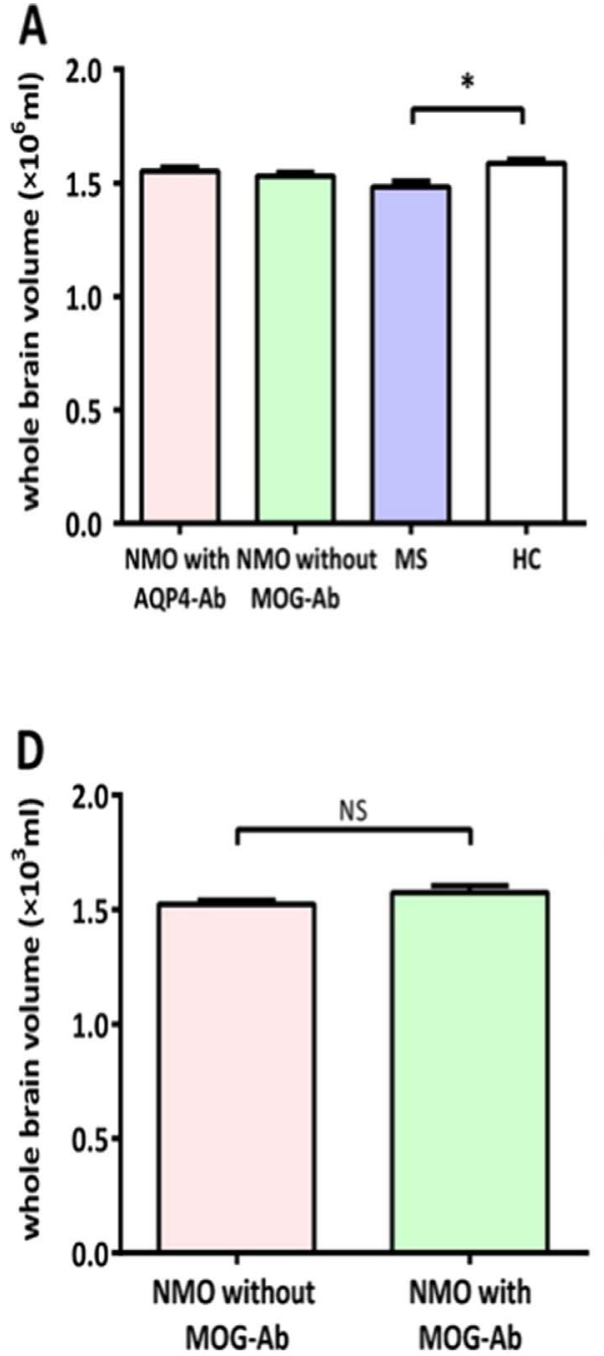
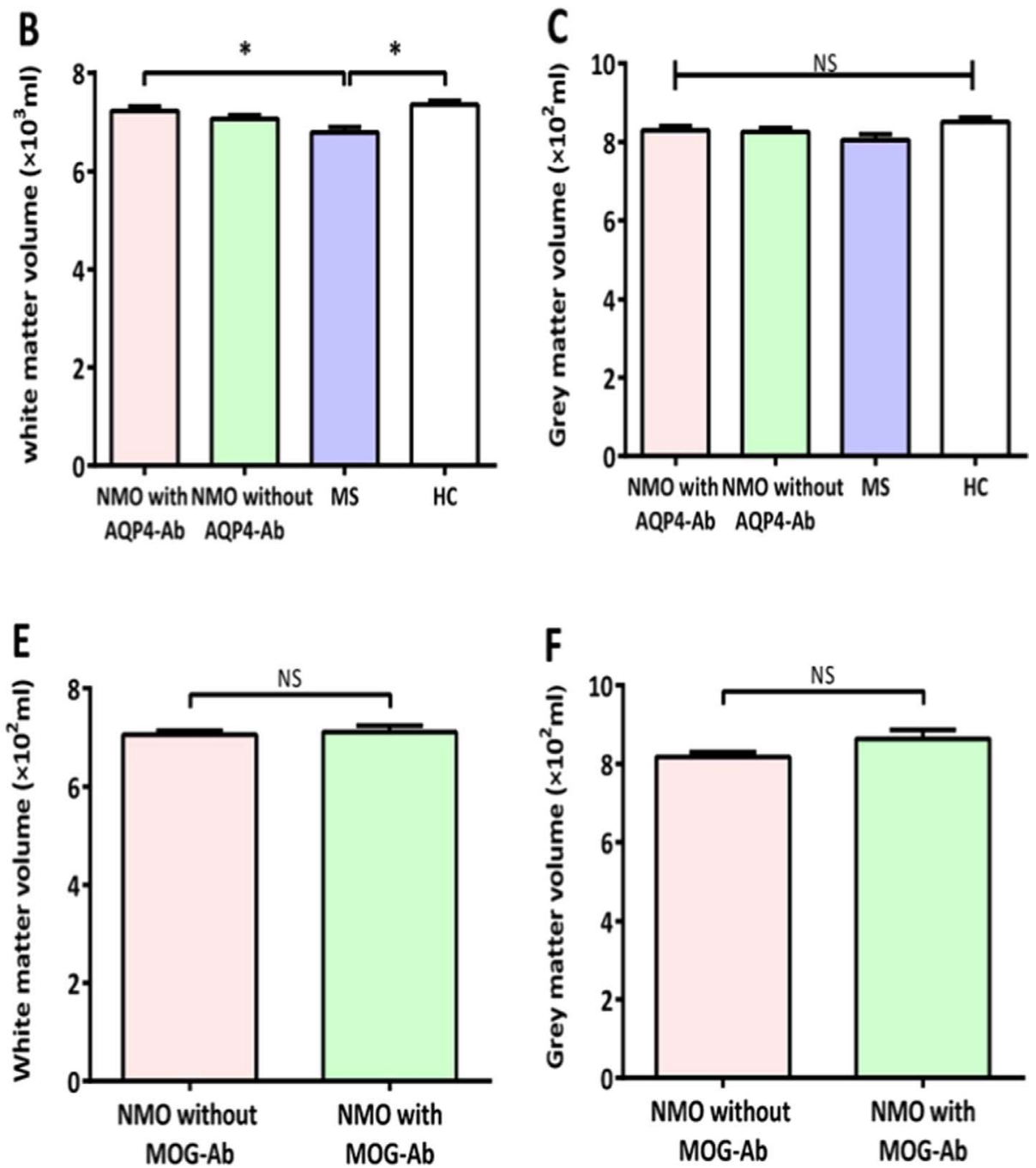

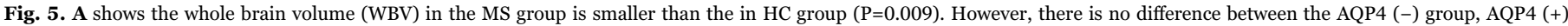

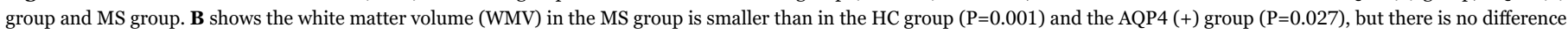

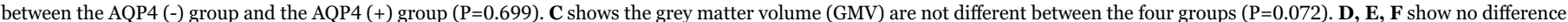
between the MOG $(+)$ group and the MOG $(-)$ group in WBV $(\mathrm{P}=0.266)$, WMV $(\mathrm{P}=0.941)$ and GMV $(\mathrm{P}=0.120)$.

(-) patients in FA (p=0.089) and MD (p=0.212) showed (Fig. $7 \mathrm{C}$ and D).

\section{Discussion}

In this study, we investigated the brain and spinal cord MRI characteristics in NMO patients, and searched for differences between AQP4-seropositive and -seronegative NMO patients. Furthermore we compared the MOG (+) and MOG (-) patients of the AQP4 (-) NMO group. We demonstrated that (1) the incidence of brain lesions and infratentorial lesions were significantly higher in the AQP4 (-) patients than in the AQP4 (+) patients. The prevalence of brain lesions in seronegative NMO cases was similar to MS cases. (2) Total spinal cord lesion length was higher in AQP4 (+) patients compared to MS patients, but no significant differences were identified between AQP4 $(+)$ and (-) or between AQP4 (-) and MS. (3) Deep grey matter atrophy including in the thalamus and hippocampus was predominantly found in AQP4 (-) patients. Whole brain atrophy was subtle in both AQP4 (+) and (-) patients. (4) Whole brain white matter diffusion was not significantly altered in both AQP4 (+) and (-) patients. (5) There were no significant differences of MRI characteristics including lesions, brain volume and white matter integrity between MOG (+) and MOG (-) patients.
Previous studies have reported that brain abnormalities are seen in the majority of NMO cases. Brain lesions are present in about half of patients at presentation and increase with disease progression (Cabrera-Gomez and Kister, 2012; Kiyat-Atamer et al., 2013; Pittock et al., 2006). The current study demonstrated that the incidence of brain lesions in NMO cases is $60 \%$, of which the incidence was $46.7 \%$ in seropositive NMO cases and $76 \%$ in seronegative NMO cases. MS patients demonstrated even higher brain lesion prevalence (92\%). This finding is consistent with previous studies(Asgari et al., 2013; Jarius et al., 2012). Infratentorial (including the cerebellum and brain stem) lesions have seldom been compared between NMO and MS. Our study has shown that the incidence of infratentorial lesions in AQP4 (-) patients is similar to MS cases. Interestingly, none of the AQP4 (+) cases in our cohort haveinfratentorial lesions.

Longitudinally extensive transverse myelitis (LETM) is an MRI feature of NMO, which is predominantly central in position and involves grey as well as white matter (Downer et al., 2012; Nakamura et al., 2008; Zhong et al., 2012). In an attempt to shed light on the difference of spinal cord lesions between AQP4 (+) and AQP4 (-) cases, we examined the number of spinal cord lesions and the total extent of spinal cord lesions in each patient. We found that there were no significant differences in the number of spinal cord lesion between the three groups. The AQP4 (+) group had longer spinal cord 
A

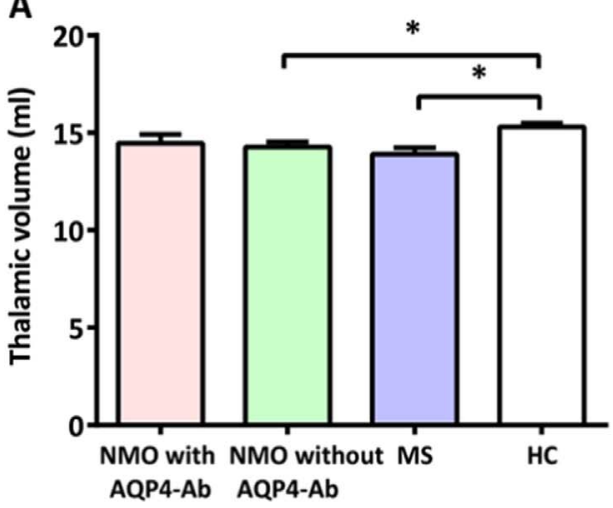

C

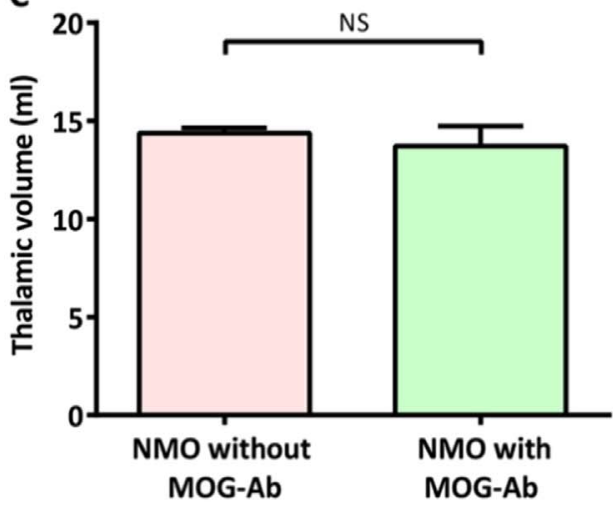

B

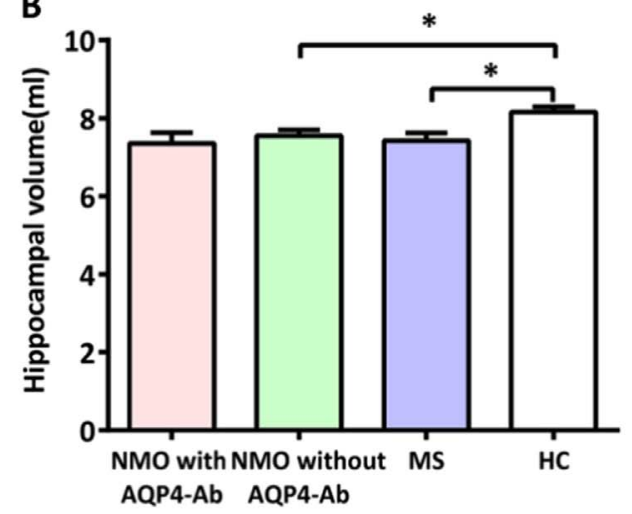

D

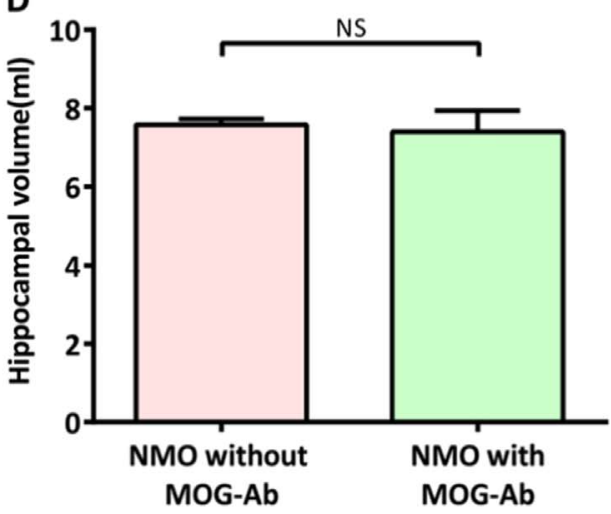

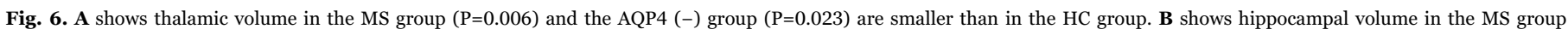

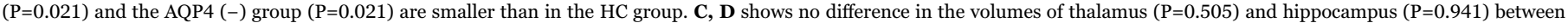
the MOG (+) group and the MOG (-) group.

lesions than the MS group, but a similar total lesion length compared with the AQP4 (-) group. The length of spinal cord lesions was higher in the AQP4 (+) group than in the MS group. The difference in brain lesions, especially ITL lesions, but not in spinal cord lesions between AQP4 (+) and (-) patients implies that brain lesions are key features differentiating AQP4 (+) vs (-).

As far as we know, progressive development of brain atrophy is a well-known feature in MS (Duan et al., 2012; Schwarz et al., 2001; Liu et al., 2015), our finding agrees with the results that the volumes of the whole brain, grey matter and white matter are reduced in MS patients compared to HC. In AQP4-seronegative NMO patients, only some deep grey matter atrophy was identified, including the thalamus and hippocampus, in the present study, suggesting that AQP4 (-) NMO patients share some common pattern of pathological mechanism causing brain damage.

In this study, we evaluated the global white matter changes of patients by measuring FA and MD across the whole brain using TBSS. Rueda Lopes et al. (2012) and Liu et al. (2012) have reported that there is extensive white matter damage in patients with NMO. However, we did not find changes in WM diffusion in NMO patients in our study, which may be due to the fact that we only measured DTI parameters of the whole brain, while previous studies confined their measurements to specific white matter tracts such optic radiation or corticospinal tract. These findings indicate that white matter injury in NMO is subtle, and no differences in diffusion metrics were identified between AQP4 (+) and $(-)$.

Experimental studies have increasingly indicate that MOG antibodies can be found in patients with NMO (Kitley et al., 2014; Mader et al., 2011; Probstel et al., 2015; Willison and Linington, 2012). Previous studies imply that MOG serostatus is another important biomarker for NMO (Kitley et al., 2014; Willison and Linington, 2012). In order to demonstrate the clinical and imaging characteristics of NMO, we tested MOG antibodies in all patients and healthy individuals. In our study, most MOG-seropositive cases come from patients with an AQP4-seronegative NMO phenotype. And our data confirmed several studies demonstrating MOG-seropositive patients tend to be males (Kitley et al., 2014; Probstel et al., 2015). Our study did not find any differences between MOG-seropositive and MOGseronegative patients in terms of MRI characteristics.

Our study has several limitations. First, this is a cross-sectional study using structural MRI measurements and the sample size is relatively small. The current results warrant further validation by large sample studies. Second, our research is mainly focused on whole brain imaging changes of patients, rather than concentrating on specific regions of interest or white matter tracts. Finally, this study only investigated the structural MRI measurement in patients. Future studies with fMRI techniques are needed to extend our findings by combining functional and structural measures.

\section{Conclusion}

The NMO patients without AQP4 antibodies showed a higher prevalence of brain lesions, infratentorial lesions, and similar spinal cord lesion length, compared to AQP4 (+) patients, and demonstrated deep grey matter atrophy without significant whole brain atrophy and diffusion alterations compared to HCs, suggesting an intermediate phenotype between typical MS and NMO. No significant differences of MRI characteristics were identified between patients with different MOG serostatus in AQP4 (-) NMO patients.

\section{Conflict of interest/role of funding source}

This study was supported by the ECTRIMS-MAGNIMS Fellowship from The European Committee for Treatment and Research in Multiple 
A

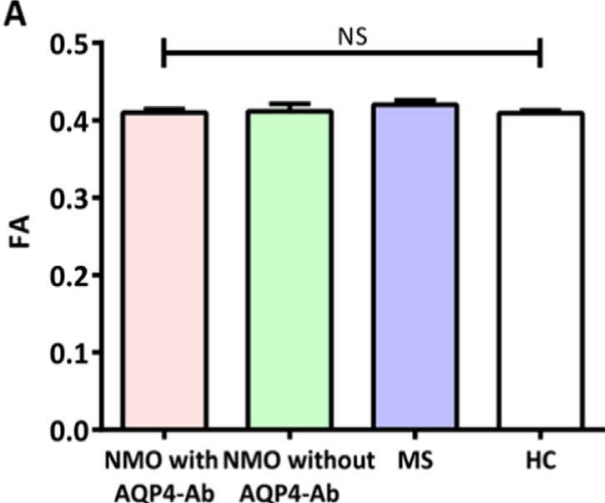

C

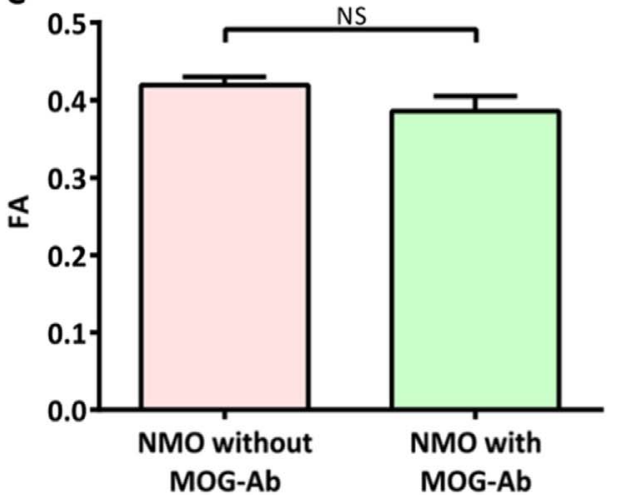

B

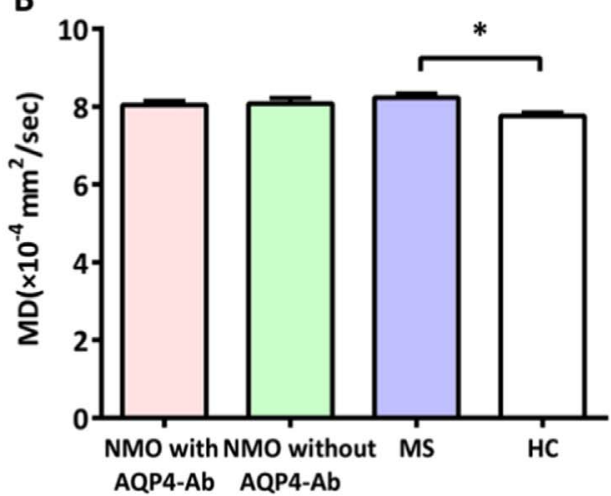

D

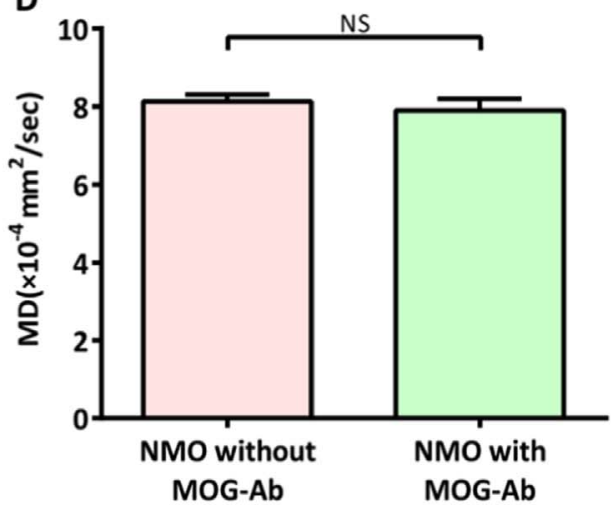

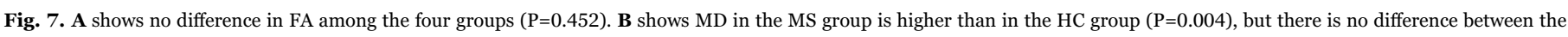

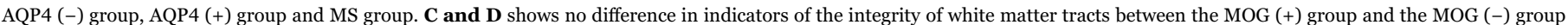
$(\mathrm{P}=0.089)(\mathrm{P}=0.212)$.

Sclerosis (ECTRIMS) (YL), the National Science Foundation of China (No. 81571631), the Beijing Natural Science fund (No. 7162077), and the Beijing Nova Programme (xx2013045). The administration of the study and data analysis was done without any input from any Biogen Idec representative. There are no other conflicts to report for any author.

\section{Acknowledgements}

This work was supported by the ECTRIMS-MAGNIMS Fellowship from The European Committee for Treatment and Research in Multiple Sclerosis (ECTRIMS) (YL), the National Science Foundation of China (No. 81571631), the Beijing Natural Science Fund (No. 7162077), and the Beijing Nova Programme (xx2013045).

\section{References}

Asgari, N., Skejoe, H.P., Lillevang, S.T., Steenstrup, T., Stenager, E., Kyvik, K.O., 2013. Modifications of longitudinally extensive transverse myelitis and brainstem lesions in the course of neuromyelitis optica (NMO): a population-based, descriptive study. BMC Neurol. 13, 33.

Cabrera-Gomez, J.A., Bonnan, M., Gonzalez-Quevedo, A., Saiz-Hinarejos, A., Marignier, R., Olindo, S., et al., 2009. Neuromyelitis optica positive antibodies confer a worse course in relapsing-neuromyelitis optica in Cuba and French West Indies. Mult. Scler. 15, 828-833.

Cabrera-Gomez, J.A., Kister, I., 2012. Conventional brain MRI in neuromyelitis optica. Eur. J. Neurol. 19, 812-819.

Chanson, J.B., Lamy, J., Rousseau, F., Blanc, F., Collongues, N., Fleury, M., et al., 2013. White matter volume is decreased in the brain of patients with neuromyelitis optica. Eur. J. Neurol. 20, 361-367.

Downer, J.J., Leite, M.I., Carter, R., Palace, J., Kuker, W., Quaghebeur, G., 2012 Diagnosis of neuromyelitis optica (NMO) spectrum disorders: is MRI obsolete? Neuroradiology 54, 279-285.

Duan, Y., Liu, Y, Liang, P., Jia, X, Yu, C. Qin, W., et al, 2012. Comparison of grey matter atrophy between patients with neuromyelitis optica and multiple sclerosis: a voxel-based morphometry study. Eur. J. Radiol. 81, e110-e114.
Jarius, S., Ruprecht, K., Wildemann, B., Kuempfel, T., Ringelstein, M., Geis, C., et al., 2012. Contrasting disease patterns in seropositive and seronegative neuromyelitis optica: a multicentre study of 175 patients. J. Neuroinflamm. 9, 14

Kimura, M.C., Doring, T.M., Rueda, F.C., Tukamoto, G., Gasparetto, E.L., 2014. In vivo assessment of white matter damage in neuromyelitis optica: a diffusion tensor and diffusion kurtosis MR imaging study. J. Neurol. Sci. 345, 172-175.

Kitley, J., Woodhall, M., Waters, P., Leite, M.I., Devenney, E., Craig, J., et al., 2012. Myelin-oligodendrocyte glycoprotein antibodies in adults with a neuromyelitis optica phenotype. Neurology 79, 1273-1277.

Kitley, J., Waters, P., Woodhall, M., Leite, M.I., Murchison, A., George, J., et al., 2014. Neuromyelitis optica spectrum disorders with aquaporin-4 and myelinoligodendrocyte glycoprotein antibodies: a comparative study. JAMA Neurol. 71, 276-283.

Kiyat-Atamer, A., Ekizoglu, E., Tuzun, E., Kurtuncu, M., Shugaiv, E., Akman-Demir, G., et al., 2013. Long-term MRI findings in neuromyelitis optica: seropositive versus seronegative patients. Eur. J. Neurol. 20, 781-787.

Lennon, V.A., Wingerchuk, D.M., Kryzer, T.J., Pittock, S.J., Lucchinetti, C.F., Fujihara, K., et al., 2004. A serum autoantibody marker of neuromyelitis optica: distinction from multiple sclerosis. Lancet 364, 2106-2112.

Liu, Y., Duan, Y., He, Y., Yu, C., Wang, J., Huang, J., et al., 2012. A tract-based diffusion study of cerebral white matter in neuromyelitis optica reveals widespread pathological alterations. Mult. Scler. 18, 1013-1021.

Liu, Y., Han, Y., Duan, Y., Huang, J., Ren, Z., Butzkueven, H., et al., 2013. Antiaquaporin-4 antibody positivity in neuromyelitis optica is associated with lesion activity. Eur. Neurol. 70, 113-116.

Liu, Y., Wang, J., Daams, M., Weiler, F., Hahn, H.K., Duan, Y., et al., 2015. Differential patterns of spinal cord and brain atrophy in NMO and MS. Neurology 84, $1465-1472$.

Mader, S., Gredler, V., Schanda, K., Rostasy, K., Dujmovic, I., Pfaller, K., et al., 2011. Complement activating antibodies to myelin oligodendrocyte glycoprotein in neuromyelitis optica and related disorders. J. Neuroinflamm. 8, 184.

Matsushita, T., Isobe, N., Piao, H., Matsuoka, T., Ishizu, T., Doi, H., et al., 2010. Reappraisal of brain MRI features in patients with multiple sclerosis and neuromyelitis optica according to anti-aquaporin-4 antibody status. J. Neurol. Sci. 291, 37-43.

Nakamura, M., Miyazawa, I., Fujihara, K., Nakashima, I., Misu, T., Watanabe, S., et al., 2008. Preferential spinal central gray matter involvement in neuromyelitis optica. An MRI study. J. Neurol. 255, 163-170.

Patenaude, B., Smith, S.M., Kennedy, D.N., Jenkinson, M., 2011. A Bayesian model of shape and appearance for subcortical brain segmentation. NeuroImage 56, 907-922.

Pichiecchio, A., Tavazzi, E., Poloni, G., Ponzio, M., Palesi, F., Pasin, M., et al., 2012. Advanced magnetic resonance imaging of neuromyelitis optica: a multiparametric 
approach. Mult Scler. 18, 817-824.

Pittock, S.J., Lennon, V.A., Krecke, K., Wingerchuk, D.M., Lucchinetti, C.F. Weinshenker, B.G., 2006. Brain abnormalities in neuromyelitis optica. Arch. Neurol. 63, 390-396.

Polman, C.H., Reingold, S.C., Edan, G., Filippi, M., Hartung, H.P., Kappos, L., et al. 2005. Diagnostic criteria for multiple sclerosis: 2005 revisions to the "McDonald Criteria". Ann. Neurol. 58, 840-846.

Probstel, A.K., Rudolf, G., Dornmair, K., Collongues, N., Chanson, J.B., Sanderson, N.S., et al., 2015. Anti-MOG antibodies are present in a subgroup of patients with a neuromyelitis optica phenotype. J. Neuroinflamm. 12, 46.

Ramanathan, S., Prelog, K., Barnes, E.H., Tantsis, E.M., Reddel, S.W., Henderson, A.P., et al., 2016. Radiological differentiation of optic neuritis with myelin oligodendrocyte glycoprotein antibodies, aquaporin-4 antibodies, and multiple sclerosis. Mult. Scler., $470-482$.

Rueda Lopes, F.C., Doring, T., Martins, C., Cabral, F.C., Malfetano, F.R., Pereira, V.C. et al., 2012. The role of demyelination in neuromyelitis optica damage: diffusiontensor MR imaging study. Radiology 263, 235-242.

Schwarz, S., Mohr, A., Knauth, M., Wildemann, B., Storch-Hagenlocher, B., 2001. Acute disseminated encephalomyelitis: a follow-up study of 40 adult patients. Neurology $56,1313-1318$.

Smith, S.M., Zhang, Y., Jenkinson, M., Chen, J., Matthews, P.M., Federico, A., et al., 2002. Accurate, robust, and automated longitudinal and cross-sectional brain change analysis. NeuroImage 17, 479-489.
Weinshenker, B.G., Wingerchuk, D.M., Pittock, S.J., Lucchinetti, C.F., Lennon, V.A., 2006. NMO-IgG: a specific biomarker for neuromyelitis optica. Dis. Markers 22, 197-206.

Willison, H.J., Linington, C., 2012. Antibodies to MOG in NMO: a seasoned veteran finds a new role. Neurology 79, 1198-1199.

Wingerchuk, D.M., Lennon, V.A., Pittock, S.J., Lucchinetti, C.F., Weinshenker, B.G., 2006. Revised diagnostic criteria for neuromyelitis optica. Neurology 66 , $1485-1489$.

Wingerchuk, D.M., Lennon, V.A., Lucchinetti, C.F., et al., 2007. The spectrum of neuromyelitis optica. Lancet Neurol. 6, 805-815.

Yang, C.S., Zhang, D.Q., Wang, J.H., Jin, W.N., Li, M.S., Liu, J., et al., 2014. Clinical features and sera anti-aquaporin 4 antibody positivity in patients with demyelinating disorders of the central nervous system from Tianjin, China. CNS Neurosci. Ther. 20, 32-39.

Zhang, L., Wu, A., Zhang, B., Chen, S., Men, X., Lin, Y., et al., 2014. Comparison of deep gray matter lesions on magnetic resonance imaging among adults with acute disseminated encephalomyelitis, multiple sclerosis, and neuromyelitis optica. Mult. Scler. 20, 418-423.

Zhang, N., Li, Y.J., Fu, Y., Shao, J.H., Luo, L.L., Yang, L., et al., 2015. Cognitive impairment in Chinese neuromyelitis optica. Mult. Scler. 21, 1839-1846.

Zhong, X.N., Wang, H.H., Bao, J., Li, R., Long, Y.M., Lu, Z.Q., et al., 2012. Relationship between neuromyelitis optica-IgG status and spinal cord magnetic resonance imaging in patients with neuromyelitis optica. Chin. Med. J. 125, 270-274. 